\title{
Rapid Voltage Changes in Power System Networks and their Effect on Flicker
}

\author{
Julio Barros, Senior Member, IEEE, Jose Julio Gutiérrez, Matilde de Apráiz, Purificación Saiz, Ramón \\ I. Diego, Senior Member, IEEE, and Andoni Lazkano, Member, IEEE
}

\begin{abstract}
A rapid voltage change (RVC) is a type of power quality disturbance that has not received sufficient attention in the international power quality standards. At present, IEC 61000-4-30, Ed. 3, defines a precise method for RVC detection and analysis. RVCs can cause or contribute to flicker. However, the existing studies on the subject have not found a correlation between the short-term flicker severity index, Pst, and the parameters of a RVC event. This paper provides an interpretation of this lack of correlation and shows that instead there is a correlation of RVC events with the instantaneous flicker sensation, $P_{\text {inst }}$. The work is based on the analysis of both simulated waveforms containing RVCs and waveforms recorded in low-voltage and medium-voltage locations.
\end{abstract}

\section{Index Terms--Flicker; Power quality; Rapid voltage changes}

\section{INTRODUCTION}

A rapid voltage change (RVC) is a type of voltage fluctuation defined as a quick transition in r.m.s. voltage occurring between two steady-state conditions, and during which the r.m.s. voltage does not exceed the dip/swell thresholds [1]. RVCs even within the normal operation voltage tolerances, are considered a disturbing phenomenon, which should be limited due to their effect on flicker, and to assure the correct performance of the power system networks [2].

There are different indices and measures to describe voltage fluctuations and their effect on flicker. Some works study the diagnostic capabilities of several voltage fluctuation indices, although these works are not focused specifically on the use of these indices to characterize the RVCs [3, 4]. With the increasing penetration of distributed energy resources, an increasing number of RVCs are expected, most of them resulting in temporary overvoltages that should be documented and analyzed. At present there are few studies reporting indices to measure RVCs in power system networks. Bollen et al [5] propose the definition of indices for the measurement of voltage fluctuations in very-short time scale, which could be used for the characterization of RVCs. Brekke et al. [6] associated RVCs with annoying flicker in

This work received financial support from the Spanish Ministry of Science and Innovation, National Plan of R+D+I (2008-2011) through the grant ENE2009-13978-C02 and from the University of the Basque Country UPV/EHU through the project UFI11/16.

J. Barros, M. de Apraiz and R. I. Diego are with the Department of Computer Science and Electronics, University of Cantabria, 39004 Santander, Spain (e-mail: julio.barros@unican.es, apraizma@gmail.com, ramon.diego@unican.es).

J. J. Gutierrez, P. Saiz and A. Lazkano are with the Department of Communications Engineering, University of the Basque Country (UPV/EHU), 48013 Bilbao, Spain (e-mail: josejulio.gutierrez@ehu.es, puri.saiz@ehu.es, andoni.lazkano@ehu.es). order to establish minimum requirements for the phenomenon. The final report of the working group C4.108 of the International Council on Large Electric Systems (CIGRE) concluded that RVCs lacked clear definition and that there were no standardized methods for their measurement [7]. In the face of this conclusion, the edition 3 of the IEC 61000-430 standard includes a precise method for detection and evaluation of these voltage events [1].

This paper reviews the definitions and regulations of RVCs, as well as the recent literature that has studied RVC events and their relationship with flicker. The bibliographic study reveals that no clear correlation has been reported among the parameters that characterize these two disturbances. The paper presents experimental work based on simulated waveforms containing RVCs as well as on recordings in actual low-voltage (LV) and medium-voltage (MV) locations with the objective of finding alternative indices that properly relate the two disturbances. The method used to measure the magnitude of the RVCs is the one proposed in the standard IEC 61000-4-30, Ed. 3. The results confirm the poor correlation between the short-term flicker severity index and the magnitude of the RVC. However, the paper proposes the use of the instantaneous flicker sensation as the parameter to analyze the actual influence of the RVCs on flicker, as well as an accurate method to identify them.

\section{RVCS IN POWER SYSTEM NETWORKS}

RVCs can be caused by switching operations such as motor starting, capacitor banks switching on/off, load switching or transformer tap-changer operations. In addition, they can also be induced by sudden load variations or by power output variations from distributed energy resources such as solar or wind power systems. Fig. 1 shows examples of RVCs associated with motor starting and capacitor switching [2].

The main effect of RVCs is on flicker, but other nonflicker effect as malfunction of control systems acting on voltage angle, braking or accelerating moments from motors or impairment of electronic equipment have been reported in the literature [8].

\section{A. Planning levels for RVCs}

Limits on the number and magnitude of the RVCs vary between different power quality standards. For compatibility level, the magnitude of an RVC is limited, under normal circumstances, to $3 \%$ of nominal voltage in MV systems, however, RVCs exceeding 3\% can occur infrequently in MV public supply systems, as is reported in IEC 61000-2-12 [9]. 
IEC 61400-21 limits the number of voltage variations in a fictitious network for grid connected wind turbines, but without specifying a method for their measurement [10].

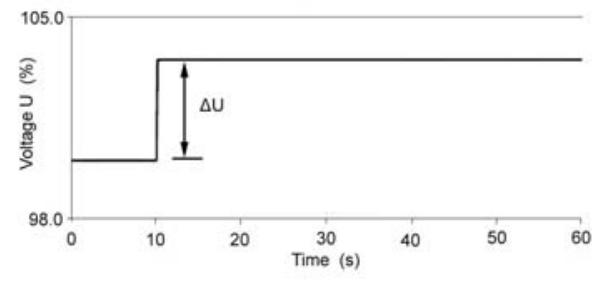

a)

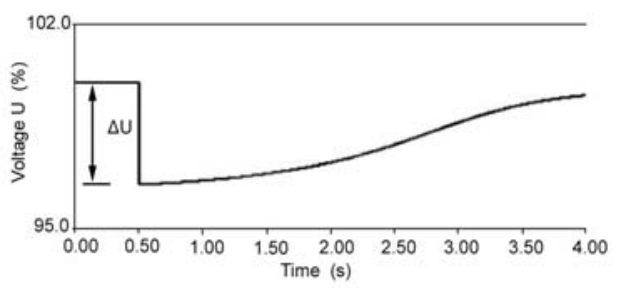

b)

Fig. 1. Examples of rapid voltage events associated with a) motor starting, b) capacitor switching [2]

Table I shows the indicative planning levels for MV, HV and EHV systems, proposed in IEC 61000-3-7 for RVCs expressed as a percentage of the nominal voltage versus the number of such changes in a given time period [2]. Table II shows the limits applied in Norway at the PCC for RVCs as a function of their magnitude and frequency for different voltage levels [6] and Table III presents a summary of practical engineering limits for RVCs in different countries.

Limits for voltage changes produced by individual pieces of equipment connected to low-voltage public grids are stipulated in standards IEC $61000-3-3$ for equipment with rated current $\leq 16 \mathrm{~A}$ [11], IEC $61000-3-11$ for the case of equipment with rated current $\leq 75 \mathrm{~A}$ [12] and IEC 61000-3-5 for equipment with rated current $>75 \mathrm{~A}$ [13].

\section{B. Detection and evaluation of RVC events}

IEC 61000-4-30, Ed. 3 proposes a precise procedure for detection and evaluation of RVC events. First, it defines the r.m.s. voltage computed over one cycle and refreshed each half-cycle, $U_{\mathrm{rms}(1 / 2)}$, as the basic calculation for the measurement and detection of RVC events. An r.m.s. voltage is in a steady-state condition if all the immediately preceding $100 \mathrm{U}_{\mathrm{rms}(1 / 2)}$ values remain within an RVC threshold from the arithmetic mean of those $100 \mathrm{U}_{\mathrm{rms}(1 / 2)}$ values. The RVC threshold is set by the user, according to the application, as a percentage of $\mathrm{U}_{\mathrm{din}}$ (it is recommended to be in the range of $1 \%$ to $6 \%$ ).

To initiate the RVC detection method, an initial set of 100 $\mathrm{U}_{\text {rms(1/2) }}$ values is recorded, computing the arithmetic mean of those values. To detect an RVC event (Fig. 2):

- A 'voltage-is-steady-state' logic signal shall be created for each voltage channel. This logic signal is true when the voltage is in steady state, and false otherwise. This logic signal is determined from the $U_{\text {rms(1/2) }}$ values of each voltage channel and it is updated for each new $U_{\text {rms(1/2) }}$ value.
TABLE I.

Indicative Planning Levels for Rapid Voltage Changes as a Function of the Number of Changes in a Given Period for MV, HV and EHV Networks [2].

\begin{tabular}{|c|c|c|}
\hline \multirow{2}{*}{$\begin{array}{c}\text { Number of changes } \\
n\end{array}$} & \multicolumn{2}{|c|}{$\Delta \mathrm{U}_{\%} / \mathrm{U}_{\mathrm{N}}$} \\
\cline { 2 - 3 } & $\mathrm{MV}$ & $\mathrm{HV} / \mathrm{EHV}$ \\
\hline $\mathrm{n} \leq 4$ per day & $5-6$ & $3-5$ \\
\hline $\begin{array}{c}\mathrm{n} \leq 2 \text { per hour } \\
\text { and }>4 \text { per day }\end{array}$ & 4 & 3 \\
\hline $2<\mathrm{n} \leq 10$ per hour & 3 & 2.5 \\
\hline
\end{tabular}

TABLE II.

Limits at the Point of Common Coupling for Rapid Voltage Changes with Respect to UN [kV] for the Respective Frequency in Norway [6].

\begin{tabular}{|c|c|c|}
\hline RVCs & \multicolumn{2}{|c|}{ Maximum frequency per 24 hours period } \\
\cline { 2 - 3 } & $0.23 \leq \mathrm{U}_{\mathrm{N}} \leq 35$ & $35<\mathrm{U}_{\mathrm{N}}$ \\
\hline$\Delta \mathrm{U}_{\text {steadystate }} \geq 3 \%$ & 24 & 12 \\
\hline$\Delta \mathrm{U}_{\mathrm{Nmax}} \geq 5 \%$ & 24 & 12 \\
\hline
\end{tabular}

TABLE III.

Summary of Practical Engineering Limits for Rapid Voltage Changes in Different Countries.

\begin{tabular}{|c|c|c|c|}
\hline Country & $\begin{array}{c}\text { Repetition Rate (r) } \\
\text { or Number of Changes }\end{array}$ & $\begin{array}{c}\text { Voltage } \\
\text { Change (\%) }\end{array}$ & $\begin{array}{l}\text { Voltage } \\
\text { Level }\end{array}$ \\
\hline USA & $\mathrm{all}^{1}$ & $3^{1}$ & not specified \\
\hline France & $\begin{array}{c}\text { all } \\
\mathrm{r}>3 / \mathrm{min}\end{array}$ & $\begin{array}{l}5 \\
2\end{array}$ & $\begin{array}{c}<400 \mathrm{kV} \\
\mathrm{MV}\end{array}$ \\
\hline England & $\mathrm{r} \geq 0.1 / \mathrm{min}$ & $\mathrm{P}_{\mathrm{st}}=0.5^{2}$ & all \\
\hline Canada & $\begin{array}{l}\text { 4/day } \\
3 / \text { day } \\
30 / \text { hr } \\
5 / \text { min }\end{array}$ & $\begin{array}{l}5 \\
4 \\
3 \\
2\end{array}$ & all \\
\hline Australia $^{3}$ & $\begin{array}{c}r \leq 4 / \text { day } \\
4 / \text { day }<r \leq 2 / \text { hour } \\
2 / \text { hour }<\text { r } \leq 10 / \text { hour }\end{array}$ & $\begin{array}{c}5-6 \\
4 \\
3\end{array}$ & $\begin{array}{l}\text { MV } \\
\text { MV } \\
\text { MV }\end{array}$ \\
\hline
\end{tabular}

${ }^{1}$ Unless $\mathrm{P}_{\mathrm{s}}=1$ curve becomes more restrictive.

${ }^{2}$ Maximum voltage change for a given repetition rate is derived from a $P_{s t}=1$ curve assuming rectangular fluctuations.

${ }^{3}$ Similar (but different) values exist for HV.

- Every time a new $U_{\text {rms(1/2) }}$ value is available, the arithmetic mean of the previous $100 \mathrm{U}_{\mathrm{rms}(1 / 2)}$ values, including the new value, is calculated.

- If every one of the previous $100 \mathrm{U}_{\mathrm{rms}(1 / 2)}$ values, including the new value, is within the RVC threshold of the arithmetic mean, then the 'voltage-is-steady-state' signal for that channel is set to true; otherwise, it is set to false.

- An RVC begins when the 'voltage-is-steady-state' logic signal changes from true to false.

- When an RVC event begins, the RVC hysteresis is applied to the RVC threshold, and changes to the "voltage-is-steadystate' logic signal are disabled for 100 half cycles.

- An RVC event ends when the 'voltage-is-steady-state' logic signal changes from false to true. When an RVC event ends, the RVC hysteresis is removed from the RVC threshold. The time stamp of the end of the RVC event is 100 half cycles prior to the logic signal changing from false to true.

If a voltage dip or voltage swell is detected during an $\mathrm{RVC}$ event, then the RVC event is discarded because the event is not an RVC.

An RVC is characterized by four parameters: start time, duration, $\Delta \mathrm{U}_{\max }$ and $\Delta \mathrm{U}_{\mathrm{ss}}$ (Fig. 2). 


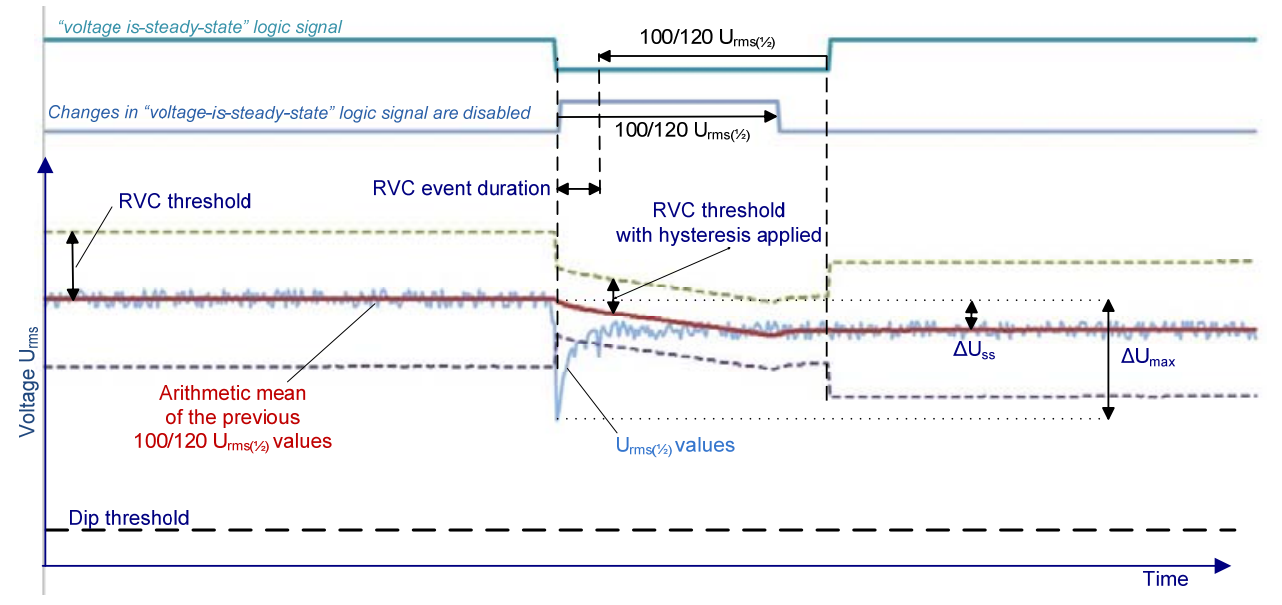

Fig.2. RVC event: example of a change in r.m.s. voltage that results in an RVC event [1].

The start time of an RVC event shall be time stamped with the time that the 'voltage-is-steady-state' logic signal became false and initiated the RVC event. The RVC event duration is 100 half cycles shorter than the length of time during which the 'voltage-is-steady-state' logic signal is false.

$\Delta \mathrm{U}_{\max }$ is the maximum absolute difference between any of the $U_{\text {rms(1/2) }}$ values during the RVC and the final arithmetic mean $100 \mathrm{U}_{\mathrm{rms}(1 / 2)}$ value just prior to the RVC. $\Delta \mathrm{U}_{\mathrm{ss}}$ is the absolute difference between the final arithmetic mean 100 $\mathrm{U}_{\text {rms }(1 / 2)}$ value prior to the $\mathrm{RVC}$ and the first arithmetic mean $100 \mathrm{U}_{\mathrm{rms}(1 / 2)}$ value after the RVC.

\section{PREVIOUS STUdiES ON THE EFFECTS OF RVCS ON FLICKER}

There is evidence that RVC events clearly affect the luminance of lighting equipment, thus causing flickering perception. However, no clear correlation has been found so far among the parameters of these two power quality disturbances [7]. The standard IEC 61000-4-15 [14] establishes the functional specifications for a flickermeter - a device that objectively quantifies the level of irritation produced by light fluctuations. The IEC flickermeter consists of five different blocks using the supply voltage as the input signal. The first four blocks model the response of the lamp-eye-brain system, producing as an output the instantaneous flicker sensation, $P_{\text {inst. }}$ The last block implements a statistical integration of $P_{\text {inst }}$ values to obtain the short-term severity, $\mathrm{P}_{\text {st }}$. More detailed descriptions of the flickermeter signal chain can be found in $[15,16]$.

Some recent papers have studied RVC events and their correlation with flicker, proposing definitions and indices for their characterization. Bollen et al. in [5] show that existing indices on RVCs may not accurately indicate their effect on flicker. The authors present a study of the correlation between the short-term flicker index $\mathrm{P}_{\text {st }}$ and the very short-voltage variation index, measured both indices in 10-minute time intervals, to conclude that there is no general correlation among them. The authors identify the relevance of the integration time and recommend the introduction of additional aggregation voltage variation indices for better performance of voltage-control equipment.

Brekke et al. in [6] presents the results of a research project carried out by the Norwegian regulator on how different parameters of a RVC event, as the magnitude of instantaneous rapid voltage changes, the rate of change $\mathrm{dv} / \mathrm{dt}$ of voltage during the event or the time duration between consecutive RVCs, affect the visibility of lighting equipment. The authors claim for the necessity to measure RVCs as a separate parameter, because measuring flicker will not ensure their correct assessment, and propose a precise definition and requirements for these events.

The report of CIGRE working group C4.108 [7] presents an assessment method of RVCs "by making use of existing definitions and measurement specifications while retaining the potential to cover the variety of practices presently in use." As in [5], the authors do not find a correlation between the short-voltage variation index and the flicker index $P_{s t}$, probably, as the authors suggest, because information that is present in one index is not present in the other. In order to capture events occurring in a very small time period, as motor starting, capacitor switching and other, the document defines a new voltage duration index, the three-second half-cycle variation index, and studies its correlation with the instantaneous flicker sensation $\mathrm{P}_{\text {inst }}$. According to the authors these two indices are poorly correlated.

As a conclusion, until present no clear relationship has been found between the parameters of RVCs and the flicker indices as defined in standard IEC 61000-4-15. There is a recent work [17] that studies the correlation between the standard flicker indices, $\mathrm{P}_{\text {st }}$ and $\mathrm{P}_{\text {inst }}$, and the parameters defined in IEC 61000-4-30, Ed.3 to characterize RVC events.

This work presented some preliminary results obtained in the measurement and analysis of RVCs in different power system networks and their effect on flicker, showing a correlation between the parameters of an RVC event and different flicker indices. 


\section{STUDY OF THE RELATIONSHIP BETWEEN RVCS AND FLICKER}

The present work is aligned with the efforts referred to in Section III to find out whether the RVC events, which cause flicker, produce identifiable variations in the classical flicker indices. In order to search for a correlation between RVCs and these flicker indexes, first RVCs of different magnitudes were analytically generated, and the corresponding values of the flicker indices were obtained. Afterwards, an equivalent study was performed with waveforms recorded in real locations: the magnitudes of the RVCs were calculated, the flicker indices were obtained, and a comparison was made between both sets of indices.

\section{A. Study with simulated RVCs}

To study the correlation between the magnitude of a RVC event and the different flicker indices, 10-min and 1-min simulated voltage waveform records of $50 \mathrm{~Hz}$ and $230 \mathrm{~V}$ r.m.s. with instantaneous RVC transitions were used. The RVC transitions, both upward and downward, were located in the mid-point of the records and their magnitude was varied from $1 \%$ to $10 \%$ of the nominal voltage in steps of $1 \%$. These voltage waveforms were used as the input signal of the IEC standard flickermeter defined in [14], computing the peak value of the instantaneous flicker sensation, $\mathrm{P}_{\text {inst, } \max }$, and the short-term flicker severity, either $\mathrm{P}_{\mathrm{st}, 10 \mathrm{~min}}$ for $10-\mathrm{min}$ waveforms or $\mathrm{P}_{\mathrm{st}, 1 \mathrm{~min}}$ for 1-min waveforms. There are several accurate implementations of the IEC flickermeter in the literature [16], [18-20]. In this work, the digital implementation described in [16] was used.

The results depicted in Fig. 3 show a clear correlation between the magnitude of an instantaneous RVC event and the flicker indices $\mathrm{P}_{\text {inst, } \max }, \mathrm{P}_{\mathrm{st}, 10 \mathrm{~min}}$ and $\mathrm{P}_{\mathrm{st}, 1 \mathrm{~min}}$. The relationship between the RVC magnitude and $P_{\text {inst,max }}$ is quadratic, whereas the relationship with $\mathrm{P}_{\mathrm{st}, 10 \mathrm{~min}}$ and $\mathrm{P}_{\mathrm{st}, 1 \mathrm{~min}}$ is linear with different slopes in the case of each index due to the different integration periods (10min vs $1 \mathrm{~min})$. In this figure, higher magnitudes of the three flicker indices are obtained in the case of an event with an upward voltage transition than in the case of a downward transition of the same magnitude. As these results contradict most of the results previously reported in the literature, it is necessary to analyze whether the observed relationship is maintained when RVCs occur in real scenarios.

\section{B. Experimental study in real scenarios}

To contrast the simulation results on the correlation of RVC events and flicker indices, this section reports some results obtained from two locations of an MV and an LV distribution network, respectively. The MV measurement point was located in a $220 / 45 \mathrm{kV}$ substation in the middlewest of Spain, close to power stations. The measurements were performed at the bus bars with phase-to-phase rated voltage $U_{\mathrm{din}}=45 \mathrm{kV}$. Fig. 4 shows the single-line diagram (SLD) of the substation where the measurement point is highlighted. The LV measurement point was located in a $230 \mathrm{~V}$ domestic line of a small town located in a steel industry area in the north of Spain in which arc furnace installations are operating. Fig. 5 shows the SLD of the $30 / 13 \mathrm{kV}$ substation that supplies the town (Fig. 5.a) as well as the $13 \mathrm{kV}$ network to the transformer substations (Fig. 5.b). The transformer substation associated to the measurement point is highlighted.

Voltage waveforms were recorded in both locations using the data acquisition system reported in [21] at different times and on different days. In the MV location data acquisition was performed at a sampling frequency of $12800 \mathrm{~S} / \mathrm{s}$, and at $6400 \mathrm{~S} / \mathrm{s}$ in the LV location. RVCs were identified and characterized applying the detection and analysis procedure defined in [1], whereas flicker indices were calculated according to the method defined in IEC 61000-4-15, Ed. 2.0 [14]. The correlation between the magnitude of the RVCs and the flicker parameters was analyzed.

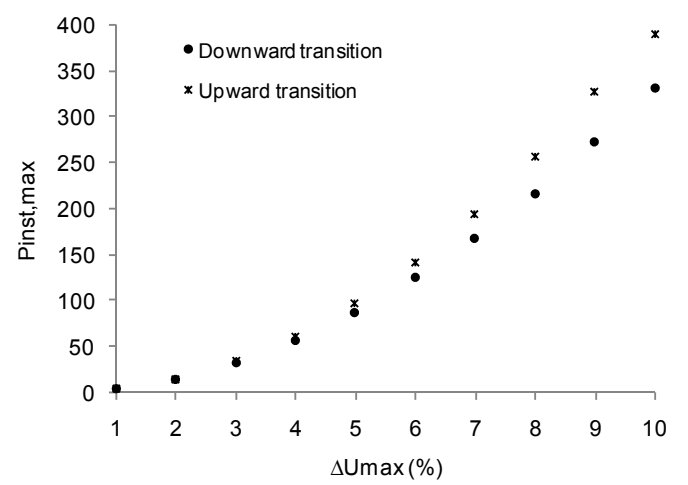

a)

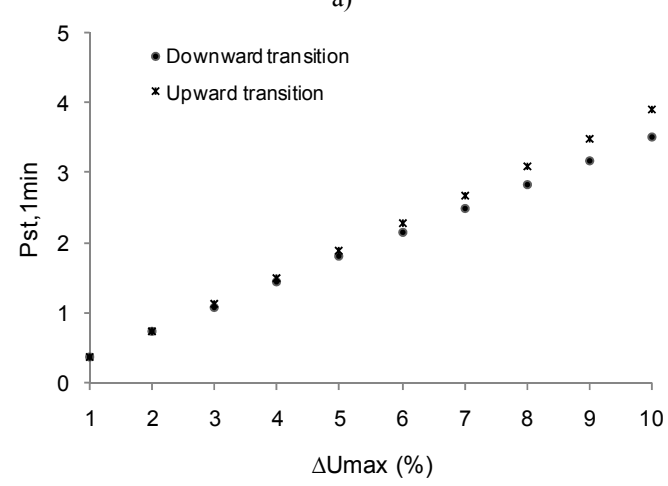

b)

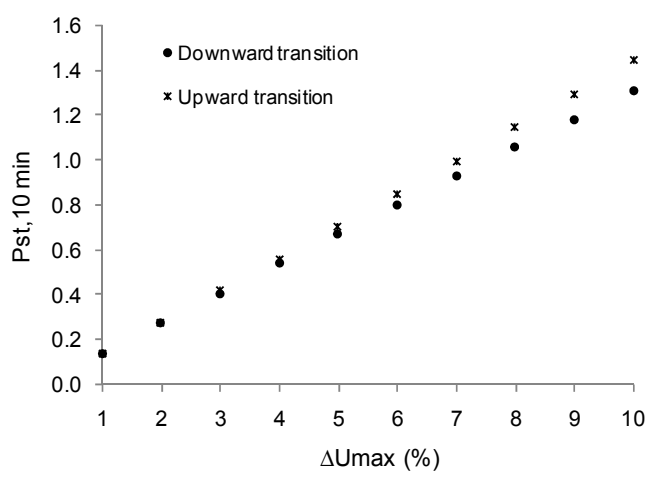

c)

Fig. 3. Correlation between RVC magnitude and flicker indices with simulated RVCs of different magnitudes a) $\mathrm{P}_{\text {inst, } \max }-\mathrm{RVC}$ magnitude, b) $\mathrm{P}_{\mathrm{st}, 1 \mathrm{~min}}-\mathrm{RVC}$ magnitude and c) $\mathrm{P}_{\mathrm{st}, 10 \mathrm{~min}}-\mathrm{RVC}$ magnitude. 


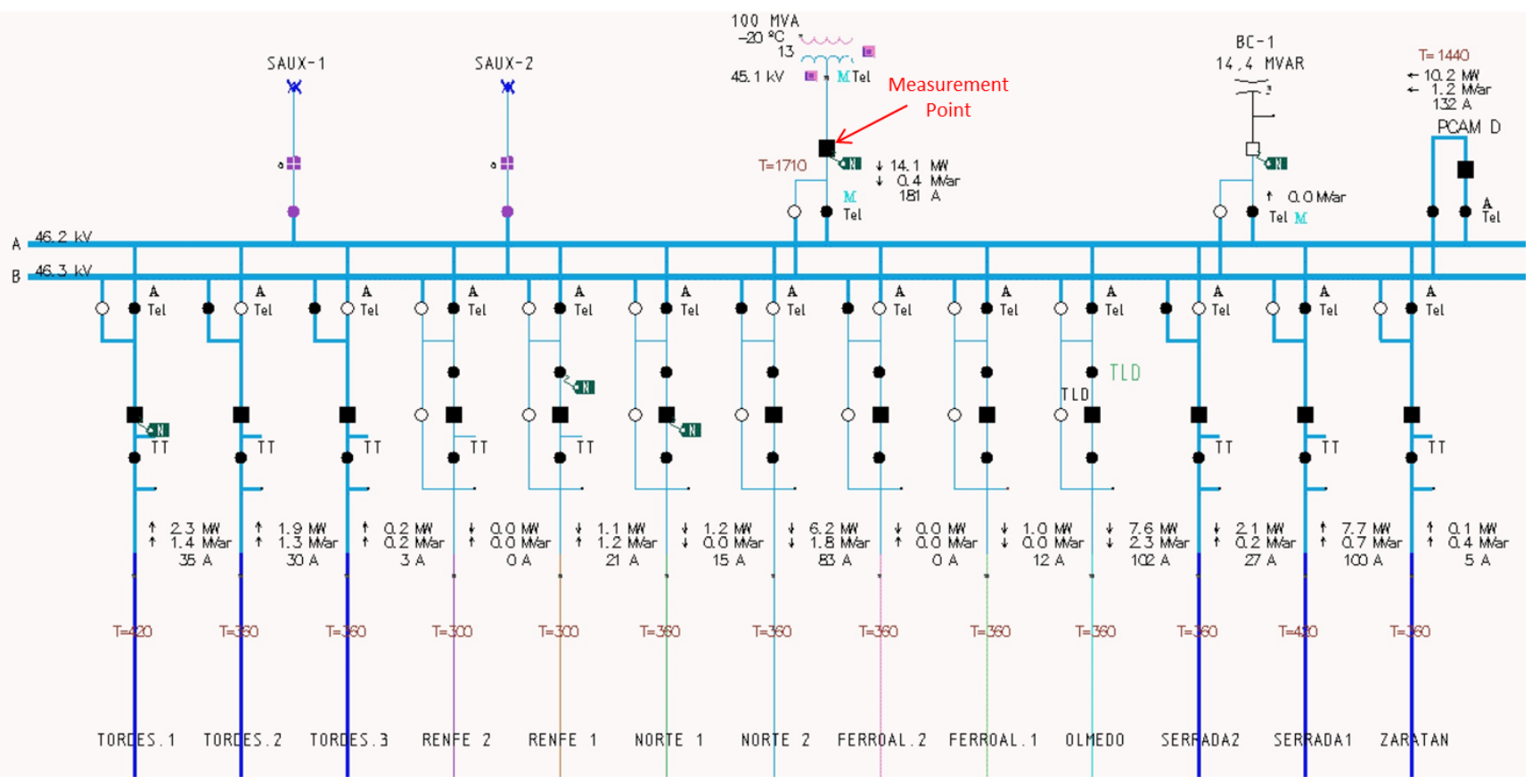

Fig. 4. Single-line diagram of the $220 / 45 \mathrm{kV}$ substation corresponding to the MV measurement location.

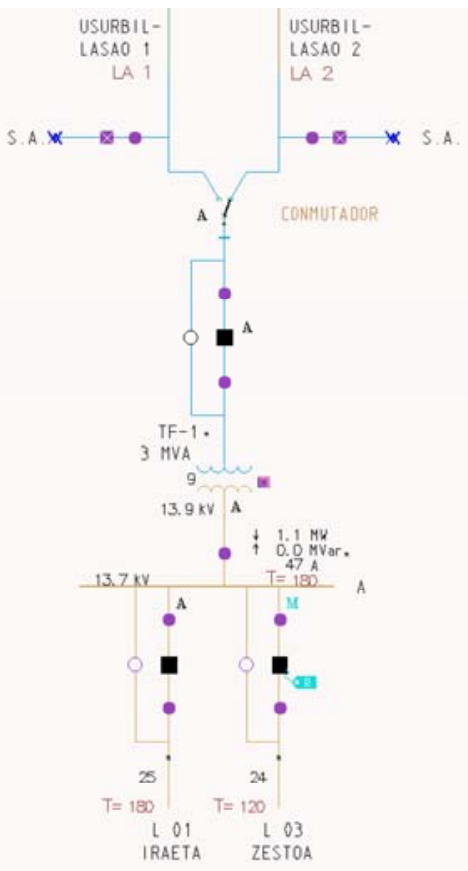

a)

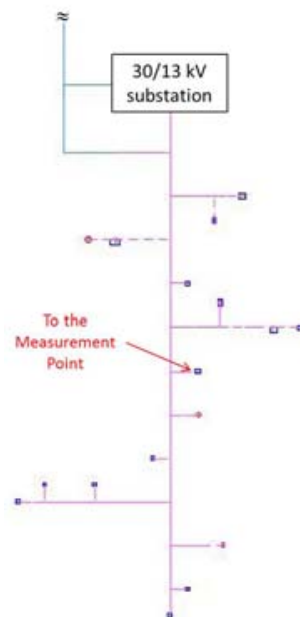

b)

Fig. 5. Single-line diagram of a) the $30 / 13 \mathrm{kV}$ substation and b) the $13 \mathrm{kV}$ network to the transformer substations, corresponding to the LV measurement location.

The $\mathrm{U}_{\mathrm{rms}(1 / 2)}$ magnitude was computed for detection of RVCs in voltage supply using different detection thresholds, from $1 \%$ to $6 \%$. In addition, the $1-$ min and 10 -min short-term flicker severity index, $P_{\text {st } 1 \text { min }}$ and $P_{\text {st } 10 \mathrm{~min}}$, and the instantaneous flicker sensation index, $\mathrm{P}_{\text {inst }}$, were computed for the different voltage records using the IEC flickermeter. Although both $\mathrm{P}_{\text {st, } 1 \text { min }}$ and $\mathrm{P}_{\text {st,10min }}$ indices were obtained, $\mathrm{P}_{\mathrm{st}, 1 \mathrm{~min}}$ has been selected for the study in the following

paragraphs so that a statistically more representative number of values was available to draw conclusions.

Fig. 6 shows the time evolution at the LV location of the

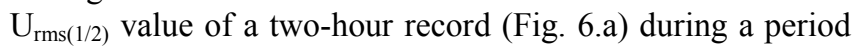
of inactivity of the arc furnaces, the "voltage-is-steady-state" logic signal for RVC detection computed using a 1\% RVC detection threshold (Fig. 6.b), the magnitude of the instantaneous flicker sensation, $P_{\text {inst }}$ (Fig. 6.c), and the magnitude of the $\mathrm{P}_{\mathrm{st}, 1 \mathrm{~min}}$ index for each 1-minute interval (Fig. 6.d). The $95^{\text {th }}$ percentile of the $\mathrm{P}_{\text {st, } 1 \text { min }}$ values for the 2-hour interval was 1.4. From the total number of $47 \mathrm{RVC}$ events detected, the 10 of them with magnitude higher than $3 \%$ of the nominal voltage are marked in Fig. 6.c. The characteristics of these events are detailed in Table IV. As can be seen, there is an exact correspondence between the start time of any of the RVC events detected during the monitoring period and a sudden variation of $\mathrm{P}_{\text {inst. }}$. Regarding the $\mathrm{P}_{\mathrm{st}, 1 \mathrm{~min}}$, a strong increase in its value is observed in the 1-min intervals where RVCs are detected.

Fig. 7 and Table V show the same information for another two-hour record in the same location of the LV network, during a period of activity of the arc furnaces. Consequently, the values of the flicker indices were higher than in the previous record, as the $95^{\text {th }}$ percentile of the $\mathrm{P}_{\text {st, } 1 \mathrm{~min}}$ summarizes with a value of 2.6. In this period 197 RVC events were detected, 17 of them with magnitude higher than $3 \%$ of nominal voltage (marked in Fig. 7.c). Observation of Fig. 7 leads to the same preliminary conclusions as Fig. 6: RVCs lead to sudden increases of both $\mathrm{P}_{\text {inst }}$ and $\mathrm{P}_{\text {st, } 1 \mathrm{~min}}$.

To study the issue further, Fig. 8 shows in a scatter diagram the flicker severity values, $\mathrm{P}_{\mathrm{st}, 1 \mathrm{~min}}$, corresponding to each of the RVC events occurring during the 4 hour period analysed in relation to same location, ordered according to the $\Delta \mathrm{U}_{\max }$ magnitude of the RVC. 


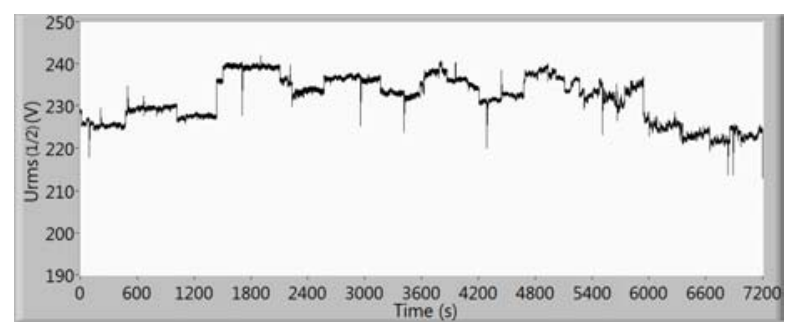

a)

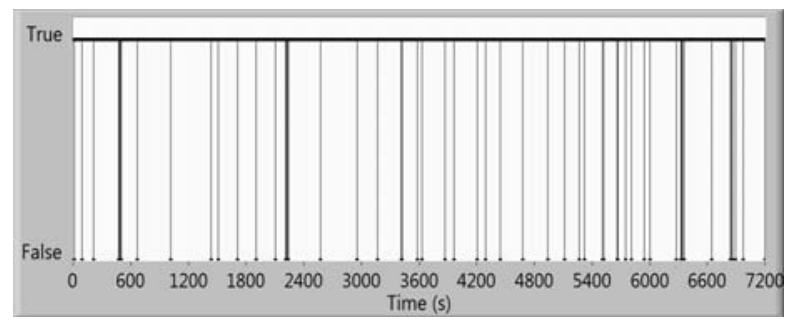

b)

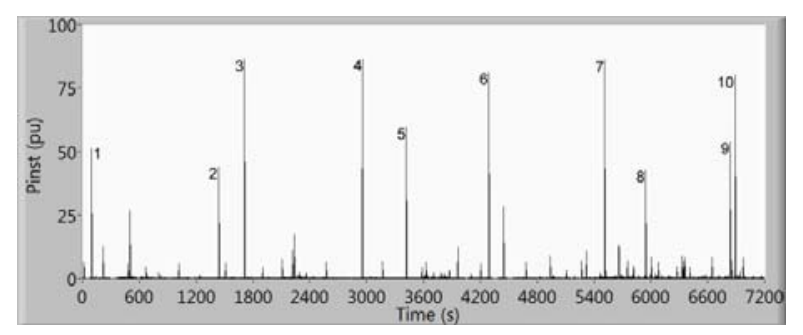

c)

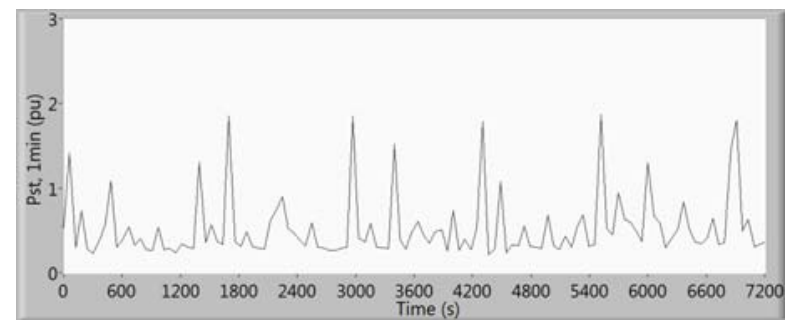

d)

Fig. 6. RVC and flicker parameters for a 2-hour record in an LV location without arc furnace activity. a) Time evolution of $U_{\mathrm{rms}(1 / 2)}$, b) steady-state logic signal computed using $1 \%$ detection threshold, c) $\mathrm{P}_{\text {inst }}$, and d) $\mathrm{P}_{\mathrm{st}, \mathrm{Imin}}$.

TABLE IV.

Characteristics of the RVC events with magnitude higher than $3 \%$ for a 2-hour record in an LV location without arc furnace activity.

\begin{tabular}{|c|c|c|c|}
\hline Event & Duration $(\mathrm{s})$ & $\Delta \mathrm{U}_{\max }(\%)$ & $\Delta \mathrm{U}_{\mathrm{ss}}(\%)$ \\
\hline 1 & 1.15 & 3.9 & 0.5 \\
\hline 2 & 0.01 & 3.8 & 3.6 \\
\hline 3 & 0.76 & 5.4 & 0.5 \\
\hline 4 & 0.79 & 5.1 & 0.5 \\
\hline 5 & 1.06 & 4.1 & 0.7 \\
\hline 6 & 0.86 & 4.9 & 0.4 \\
\hline 7 & 0.80 & 5.2 & 0.5 \\
\hline 8 & 0.01 & 3.7 & 3.6 \\
\hline 9 & 1.21 & 3.8 & 0.5 \\
\hline 10 & 0.91 & 4.7 & 0.4 \\
\hline
\end{tabular}

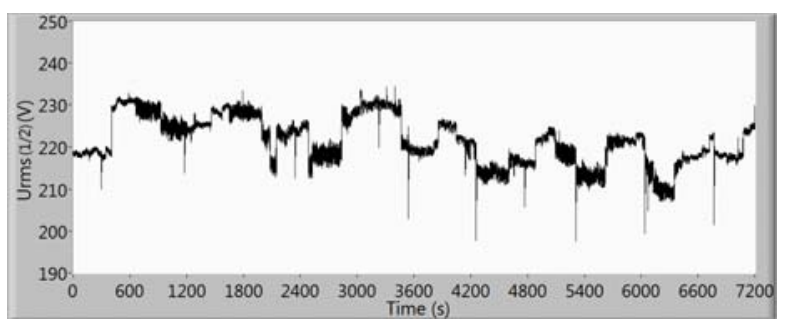

a)

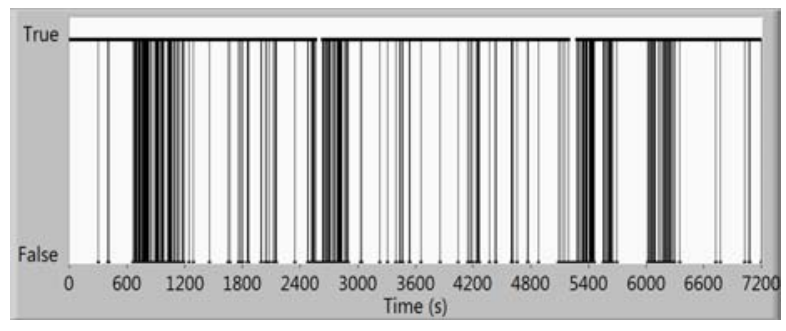

b)

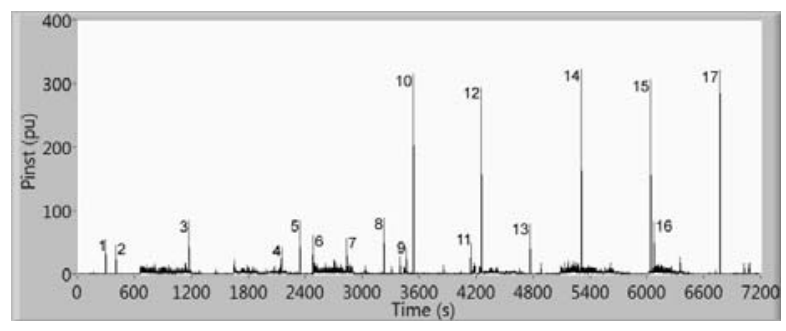

c)

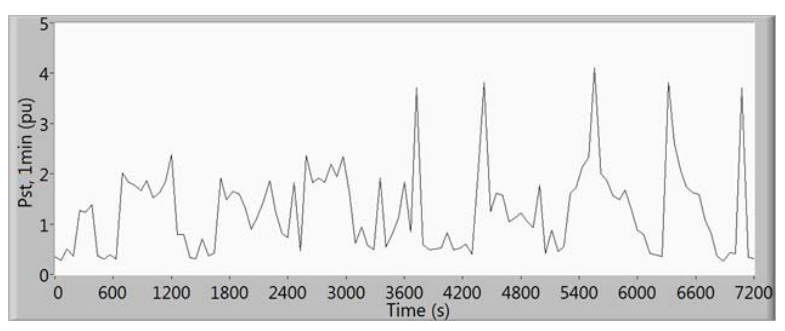

d)

Fig. 7. RVC and flicker parameters for a 2-hour record in an LV location with arc furnace activity. a) Time evolution of $U_{\operatorname{rms}(1 / 2)}$, b) steady-state logic signal computed using $1 \%$ detection threshold, c) $P_{\text {inst }}$, and d) $P_{s t, 1 \text { min }}$

TABLE V.

Characteristics of the RVC events with magnitude higher than $3 \%$ for a 2-hour record in an LV location with arc furnace activity.

\begin{tabular}{|c|c|c|c|}
\hline Event & Duration $(\mathrm{s})$ & $\Delta \mathrm{U}_{\max }(\%)$ & $\Delta \mathrm{U}_{\mathrm{ss}}(\%)$ \\
\hline 1 & 1.24 & 3.8 & 0.3 \\
\hline 2 & 0.01 & 3.6 & 3.4 \\
\hline 3 & 1.50 & 5.0 & 0.5 \\
\hline 4 & 0.12 & 3.6 & 3.1 \\
\hline 5 & 0.91 & 4.9 & 0.5 \\
\hline 6 & 0.01 & 4.1 & 3.9 \\
\hline 7 & 21.93 & 4.4 & 3.9 \\
\hline 8 & 0.87 & 5.1 & 0.5 \\
\hline 9 & 2.49 & 4.0 & 3.1 \\
\hline 10 & 0.15 & 9.2 & 0.9 \\
\hline 11 & 1.20 & 3.6 & 0.5 \\
\hline 12 & 6.70 & 9.3 & 1.9 \\
\hline 13 & 1.04 & 4.6 & 0.4 \\
\hline 14 & 10.23 & 9.0 & 1.5 \\
\hline 15 & 0.22 & 9.2 & 0.9 \\
\hline 16 & 12.44 & 5.0 & 0.6 \\
\hline 17 & 0.13 & 9.3 & 1.2 \\
\hline
\end{tabular}




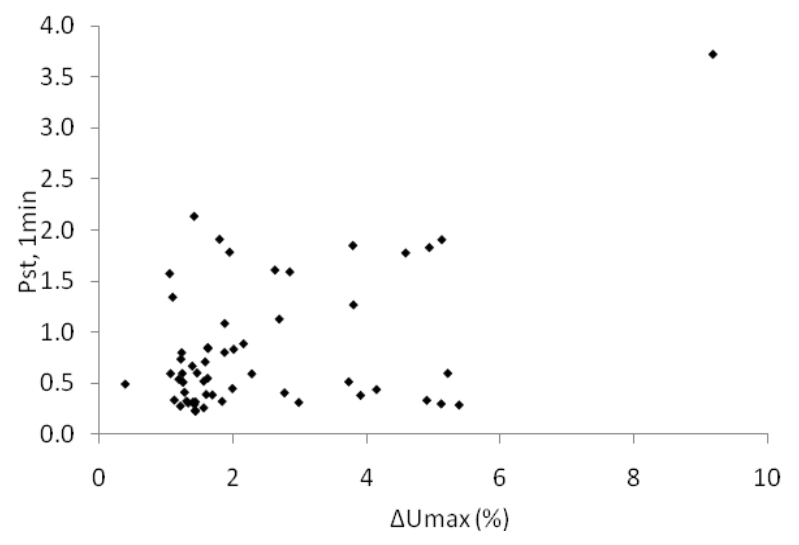

Fig. 8. Correlation $\Delta \mathrm{U}_{\max }-\mathrm{P}_{\mathrm{st}, 1 \mathrm{~min}}$ in an LV location.

Only those 1-min intervals where a single RVC event occurred are included in the scatter diagram, so there is a oneto-one relationship between a $\Delta \mathrm{U}_{\max }$ value (RVC characterization) and a $\mathrm{P}_{\text {st, } 1 \text { min }}$ value (flicker index). The general tendency of the $\mathrm{P}_{\text {st, } 1 \text { min }}$ follows that of $\Delta \mathrm{U}_{\max }$ (as the RVC magnitude increases, the flicker severity index becomes higher). This is similar to the tendency observed with simulated RVCs (Fig. 3.b). However, Fig. 8 also reveals a significant dispersion in the values: for the same $\Delta \mathrm{U}_{\max }$ value, the range of $\mathrm{P}_{\text {st, } 1 \text { min }}$ values is very wide. For example, for transitions of $\Delta \mathrm{U}_{\max } \approx 5 \%, \mathrm{P}_{\text {st, } 1 \text { min }}$ varies from 0.4 to 1.9 . Another view of this dispersion is that RVCs of different magnitudes can produce similar $\mathrm{P}_{\text {st, } 1 \text { min }}$ values. For example, a $\mathrm{P}_{\mathrm{st}, 1 \mathrm{~min}}$ value of 1.9 can be associated with RVCs whose $\Delta \mathrm{U}_{\max }$ varies approximately from $2 \%$ to $5 \%$. Thus, it is not possible to establish a relationship between the RVC magnitude and the $\mathrm{P}_{\text {st, } 1 \text { min }}$ index, confirming what previous studies had already stated [5], [7].

These discrepancies between the results obtained from the simulations and those obtained with real voltage recordings can be explained considering the effects of the temporal integration. One $\mathrm{P}_{\text {st, } 1 \mathrm{~min}}$ value is obtained as a result of a statistical calculation of the $P_{\text {inst }}$ values over the whole $1 \mathrm{~min}$ interval. In the simulated waveforms, the only disturbance that generates $\mathrm{P}_{\text {inst }}$ values different from 0 and which therefore contributes to the $\mathrm{P}_{\mathrm{st}, 1 \mathrm{~min}}$ value, is the sudden transition in the middle of the interval, i.e. the RVC. However, in the recorded waveforms there are other disturbances, apart from the RVCs, that produce non-zero $\mathrm{P}_{\text {inst }}$ and affect the $\mathrm{P}_{\text {st, } 1 \mathrm{~min}}$ to a greater or lesser extent, since they are integrated together to obtain a single value for the $1 \mathrm{~min}$ interval. Moreover, if more than one RVC with different magnitudes had been considered during the 1-min integration period, the effect of all of them would be combined to provide one single $P_{\text {st }, 1 \mathrm{~min}}$ value, which would in no case be proportional to the magnitudes of all the contributing RVCs, as previous studies stated [5], [7]. These results showed that it is not possible to detect the existence of RVCs from the analysis of the flicker severity values.

The study also required an in-depth analysis of the relationship between the RVC magnitude and the instantaneous flicker sensation, $\mathrm{P}_{\text {inst }}$. Fig. 9 shows in the top subplot the time evolution of the $U_{\operatorname{rms}(1 / 2)}$ magnitude of voltage supply during the event numbered as 1 in Fig. 6.c, and the $\mathrm{P}_{\text {inst }}$ corresponding to the same event in the bottom subplot. The $\Delta \mathrm{U}_{\max }$ of the depicted $\mathrm{RVC}$ is $3.91 \%$, and it causes a $\mathrm{P}_{\text {inst,max }}$ of $51.03 \mathrm{pu}$. This relationship is clearly similar to the results obtained from the simulated waveforms, represented in Fig. 3.a.

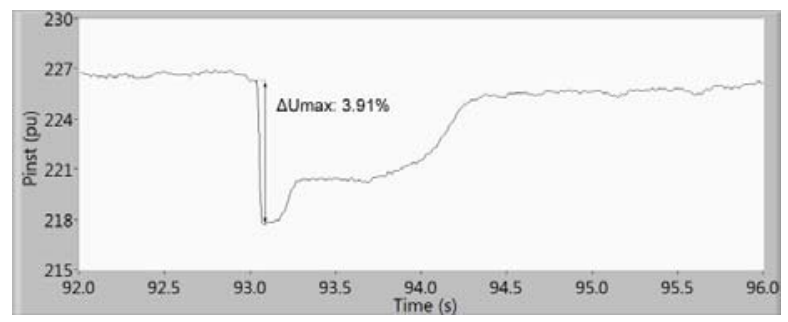

a)

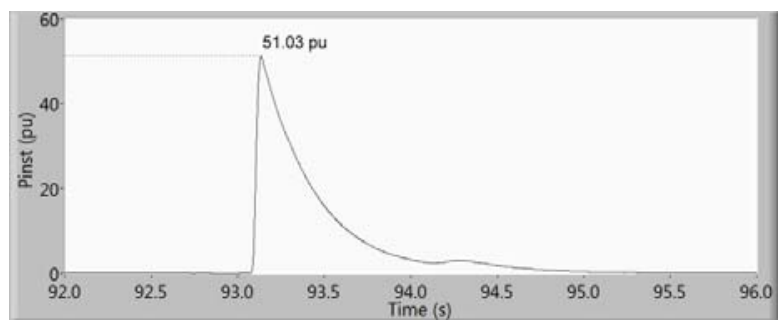

b)

Fig. 9. Detail of the RVC event numbered as 1 in Fig. 4.c. a) $U_{\text {rms(1/2) value of }}$ voltage supply, and b) time evolution of $\mathrm{P}_{\text {inst }}$ during the event.

To extend the analysis of the relationship between the RVC magnitude and $\mathrm{P}_{\text {inst }}$, Fig. 10 shows in a scatter diagram the relationship between the $\Delta \mathrm{U}_{\max }$ magnitude and the $\mathrm{P}_{\text {inst, } \max }$ values measured during each of the $244 \mathrm{RVC}$ events detected at this site. According to the behaviour observed with simulated RVCs, a distinction is made between RVCs with a downward transition (Fig. 10.a) and those with an upward transition (Fig. 10.b). The $\mathrm{P}_{\text {inst,max }}-\Delta \mathrm{U}_{\max }$ relationship of the RVCs of the LV location are shown as black markers, and the tendency as a black line. To facilitate the comparison, the results from simulated waveforms, previously reported in Fig. 3.a, are included here in red. A very close correlation with the one found for the case of simulated events has been found for this site. More specifically, a quadratic relationship has been found between the $\Delta \mathrm{U}_{\max }$ of a RVC event and the maximum value of the $\mathrm{P}_{\text {inst }}$ during the event. The average deviation between the experimental and simulated $\mathrm{P}_{\text {inst,max }}$ values for the RVC events with $\Delta \mathrm{U}_{\max }$ magnitude above $3 \%$ was $8.4 \%$.

In order to extend the validity of these results, Fig. 11 shows the time evolution for waveforms registered during 24 hours at the MV location of the $U_{\operatorname{rms}(1 / 2)}$ value (Fig. 11.a), the "voltage-is-steady-state" logic signal for RVC detection computed using a 1\% RVC detection threshold (Fig. 11.b) and the magnitude of the instantaneous flicker sensation, $\mathrm{P}_{\text {inst }}$ (Fig. 11.c). The $95^{\text {th }}$ percentile of the $\mathrm{P}_{\text {st, } 1 \text { min }}$ values for the 24hour interval was 0.3 . The frequency of occurrence of RVCs in this $\mathrm{MV}$ record is much lower than in the previous $\mathrm{LV}$ records: over the 24 hours only 9 RVC events were detected using a $1 \%$ RVC detection threshold, and only 3 of them had a magnitude higher than $3 \%$ of the nominal value. The exact correspondence between the start time of the RVC events and a sudden variation of $\mathrm{P}_{\text {inst }}$ is easily noted in the figure. 


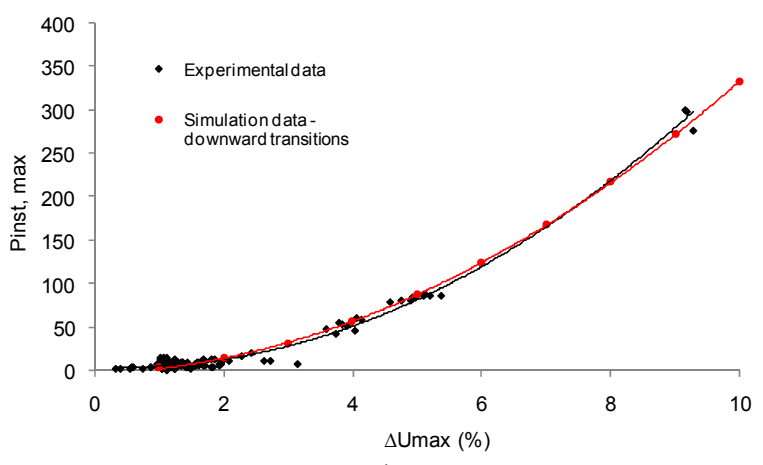

a)

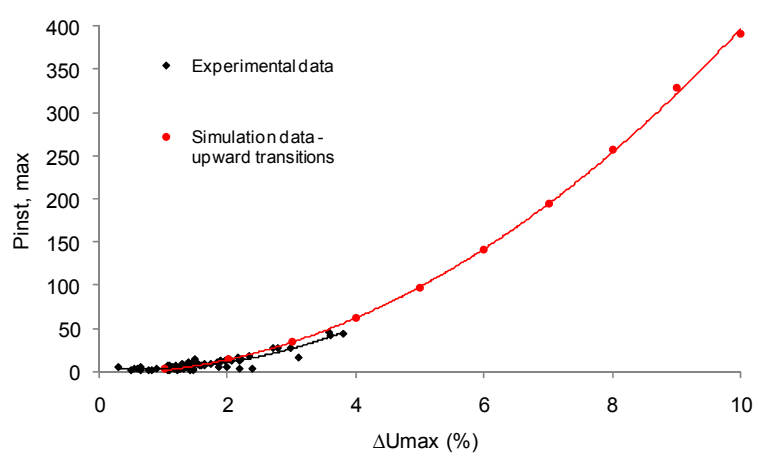

b)

Fig. 10. Correlation $\Delta \mathrm{U}_{\max }-\mathrm{P}_{\text {inst,max }}$ for the RVCs detected in an LV location. a) RVCs with downward transition, and b) RVCs with upward transition.

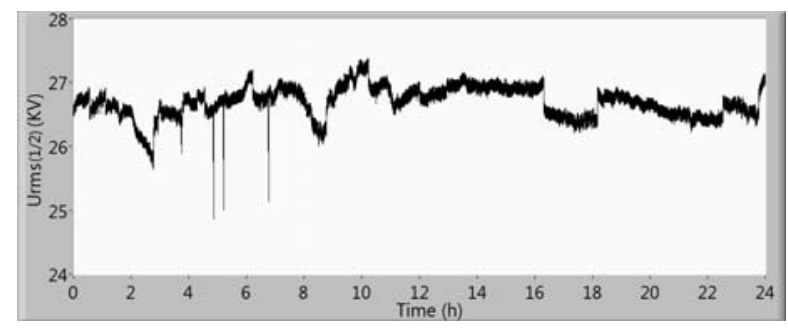

a)

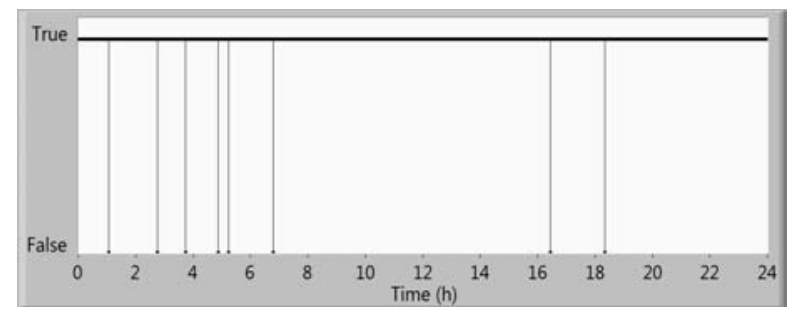

b)

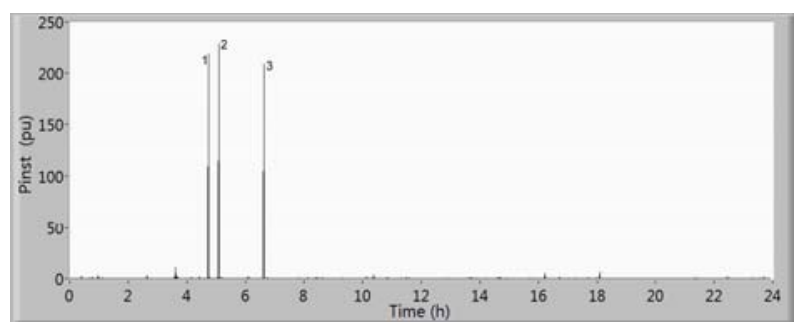

c)

Fig. 11. RVC and flicker parameters for a 24-hour record in an MV location. a) Time evolution of $U_{\mathrm{rms}(1 / 2)}$, b) steady-state logic signal computed using $1 \%$ detection threshold and c) $\mathrm{P}_{\text {inst }}$
Fig. 12 shows in a scatter diagram the relationship between the $\Delta \mathrm{U}_{\max }$ magnitude and the $\mathrm{P}_{\text {inst,max }}$ values measured during each of the RVC events detected in this MV record. In this site there is also a very close correlation with the one shown in Fig. 3.a for the case of simulated events.

Considering these results, it is possible to use the IEC flickermeter as a detector of the existence of RVCs. The instantaneous flicker sensation could be used to obtain information about the start time and the $\Delta \mathrm{U}_{\max }$ of the RVCs.

Moreover, the clear correlation between the magnitude of RVCs and $\mathrm{P}_{\text {inst }}$ enables the use of the RVC detection as a diagnosis tool in order to identify the presence of disturbing loads that could potentially produce flicker. Although the RVC detection cannot provide information about the rate of change of the disturbing load, it informs about the level of perception of the light fluctuation and the number of disturbing events.

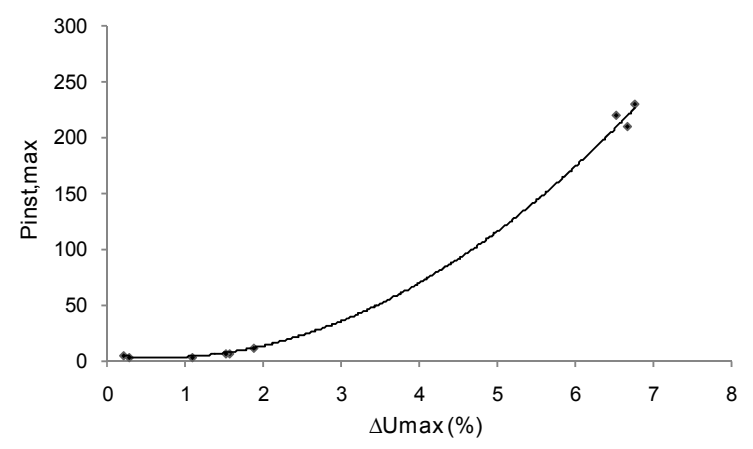

Fig. 12. Correlation between $\Delta \mathrm{U}_{\max }$ and $\mathrm{P}_{\text {inst, } \max }$ for the RVCs detected in an MV location

\section{CONCLUSION}

This paper reviews the definitions and the international regulations on RVCs and studies the relationship between those events and flicker. IEC 61000-4-30, Ed. 3 defines a precise method for RVC detection and analysis. The existing studies on the subject have not found a correlation between the short-term flicker severity index, $\mathrm{P}_{\mathrm{s}}$, and the parameters of an RVC event. This paper uses the new procedure defined in the Ed. 3 of the standard to clarify the relationship between the two disturbances. The study is performed by analyzing both simulated RVC events and RVC events detected within voltage records in Spanish LV and MV power system networks.

The results from the simulations reveal a clear correlation between the flicker parameters, $\mathrm{P}_{\mathrm{st}, 1 \mathrm{~min}}$ and $\mathrm{P}_{\mathrm{st}, 10 \mathrm{~min}}$, and the magnitude that characterizes the RVC events, $\Delta \mathrm{U}_{\text {max }}$. This conclusion contradicts the results from existing studies. However, the results from the voltage recordings in real locations reveal that the dispersion of the $\mathrm{P}_{\mathrm{st}}-\Delta \mathrm{U}_{\max }$ correlation values is much larger than in the case of simulated waveforms. The reason for this lies in the integration of several $P_{\text {inst }}$ values over a defined short-term interval that is necessary to calculate the flicker severity index, $\mathrm{P}_{\mathrm{st}}$. The existence within the same short-time interval of other disturbances affecting flicker, or the coincidence of several RVC events within the same short-time interval, impedes a 
precise correlation between $\mathrm{P}_{\mathrm{st}}$ and $\Delta \mathrm{U}_{\max }$. Hence, if there is a single RVC event within the short-time interval and this is the only disturbance affecting flicker, there is a linear relationship between $\Delta \mathrm{U}_{\max }$ of the RVC event and $\mathrm{P}_{\mathrm{st}}$. But in a general case there is a lack of correlation between $P_{\text {st }}$ and $\Delta U_{\max }$, which is the same conclusion reached by previous studies.

As a result, the $\mathrm{P}_{\mathrm{st}}$ indicator cannot be used as a parameter for analyzing the influence of RVCs on annoying flicker. Further study is needed to analyze how the flicker produced by several RVC events of different magnitudes are integrated in a single $\mathrm{P}_{\mathrm{st}}$ value, as well as how the flicker produced by the RVCs are integrated with the flicker caused by other disturbances different from RVCs.

However, the fast transition that characterizes the RVC events is reflected as a large-magnitude transient in $\mathrm{P}_{\text {inst }}$. This characteristic makes it possible to identify the influence of the RVC event on flicker through a non-integrated parameter such as $\mathrm{P}_{\text {inst,max }}$. The quadratic correlation between $\mathrm{P}_{\text {inst,max }}$ during the RVC event and $\Delta \mathrm{U}_{\max }$ obtained from simulations is clearly consistent with the results from the voltage recordings in LV and MV locations. For both scenarios, the dispersion of the correlation values is almost negligible. Furthermore, the results for the case of upward transitions and downward transitions reveal specific tendencies.

As a result, the $\mathrm{P}_{\text {inst }}$ indicator can be used as a parameter for analyzing the actual influence of RVCs on flicker. Moreover, as $\mathrm{P}_{\text {inst }}$ provides information about the start time and the $\Delta \mathrm{U}_{\max }$ of the RVCs, the $\mathrm{P}_{\text {inst }}$ output of the IEC flickermeter could be used to develop an RVC detector, thus avoiding the need of a separate tool. On the other hand, the detection of RVCs could be used to diagnose the existence of disturbing loads that affect flicker.

\section{REFERENCES}

[1] Electromagnetic Compatibility (EMC) - Part 4-30: Testing and measurement techniques. Power quality measurement methods, IEC 61000-4-30, Ed. 3, 2015.

[2] Electromagnetic Compatibility (EMC) - Part 3-7: Limits - Assessment of emission limits for the connection of fluctuating installations to $M V$, $H V$ and EHV power systems, IEC 61000-3-7, 2008.

[3] G. Wiczynski, "Analysis of Voltage Fluctuations in Power Networks," IEEE Transactions on Instrumentation and Measurement, Vol. 57, No. 11, pp. 2655-2664, November 2008.

[4] G. Wiczynski, "Diagnostic capabilities of voltage fluctuation indices a case study," IEEE $15^{\text {th }}$ International Conference on Harmonics and Quality of Power (ICHQP), Hong Kong, 2012.

[5] M. H. J. Bollen, M. Häger, and C. Schwaegerl, "Quantifying voltage variations on a time scales between 3 seconds and 10 minutes," presented at the $18^{\text {th }}$ International Conference on Electricity Distribution, CIRED 2005, Turin, Italy, 2005.
[6] K. Brekke, H. Seljeseth, and O. Mogstad, "Rapid voltage changes definition and minimum requirements," presented at the $20^{\text {th }}$ International Conference on Electricity Distribution, CIRED 2009, Prague, Czech Republic, 2009.

[7] CIGRE Working Group C4.108, "Review of Flicker Objectives for LV, MV, and HV Systems," February 2011.

[8] A. J. Schlabbach, D. Blume, T. Stephanblome, Voltage quality in electrical power systems, Institution of Electrical Engineers, London, 2001 .

[9] Electromagnetic compatibility (EMC) - Part 2-12: Environment Compatibility levels for low-frequency conducted disturbances and signalling in public medium-voltage power supply systems, IEC 610002-12, 2003.

[10] Wind turbines - Part 21: Measurement and assessment of power quality characteristics of grid connected wind turbines. IEC 61400-21, 2008.

[11] Electromagnetic Compatibility (EMC) - Part 3-3: Limits - Limitation of voltage changes, voltage fluctuations and flicker in public lowvoltage supply systems, for equipment with rated current $\leq 16 \mathrm{~A}$ per phase and not subject to conditional connection, IEC 61000-3-3, 2013.

[12] Electromagnetic compatibility (EMC) - Part 3-11: Limits - Limitation of voltage changes, voltage fluctuations and flicker in public lowvoltage supply systems - Equipment with rated current $\leq 75$ A per phase and subject to conditional connection, IEC 61000-3-11, 2000.

[13] Electromagnetic compatibility (EMC) - Part 3-5: Limits - Limitations of voltage fluctuations and flicker in low-voltage power supply systems for equipment with rated current greater than 75 A. IEC 61000-3-5, 2009 .

[14] Electromagnetic Compatibility (EMC) - Part 4-15: Testing and measurement techniques. Flickermeter, Functional and design specifications, IEC61000-4-15, 2010.

[15] G. Wiczynski, "Simple model of flickermeter signal chain for deformed modulating signals," IEEE Transactions on Power Delivery, Vol. 23, No. 4, pp. 1743-1748, October 2008.

[16] J.J. Gutierrez, L.A. Leturiondo, J. Ruiz, A. Lazkano, P. Saiz, I. Azkarate, "Effect of the Sampling Rate on the Assessment of Flicker Severity Due to Phase Jumps," IEEE Transactions on Power Delivery, Vol. 26, No. 4, pp. 2215-2222, October 2011.

[17] M. de Apraiz, J. Barros, R.I. Diego, J.J. Gutierrez, K. Redondo and I. Azcarate, "Detection and analysis of rapid voltage changes in power system networks," IEEE AMPS 2014, Aachen, Germany, 24-26 September, 2014, pp. 81-86.

[18] G. Wiczynski, "Inaccuracy of Short-Term Light Flicker $\mathrm{P}_{\text {st }}$ Indicator Measuring With a Flickermeter," IEEE Transactions on Power Delivery, Vol. 27 , No. 2, pp. 842-848, April 2012.

[19] G.W. Chang, C. Cheng-I, Y. Huang, "A Digital Implementation of Flickermeter in the Hybrid Time and Frequency Domains," IEEE Transactions on Power Delivery, Vol. 24 , No. 3, pp. 1475-1482, July 2009.

[20] A. Hooshyar, E.F. El-Saadany, "Development of a Flickermeter to Measure Non-Incandescent Lamps Flicker," IEEE Transactions on Power Delivery, Vol. 28 , No. 4, pp. 2103-2115, October 2013.

[21] J.J. Gutierrez, J. Ruiz, L. A. Leturiondo, and A. Lazkano, "Flicker Measurement System for Wind Turbine Certification," IEEE Transactions on Instrumentation and Measurement, Vol. 58, No. 2, pp. 375-382, February 2009. 